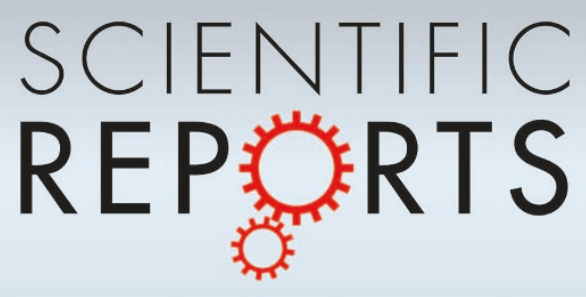

OPEN

SUBJECT AREAS:

STATISTICAL PHYSICS, THERMODYNAMICS AND NONLINEAR DYNAMICS

FLUID DYNAMICS

ASTRONOMY AND PLANETARY

SCIENCE

APPLIED PHYSICS

Received

20 June 2012

Accepted

1 November 2012

Published

15 November 2012

Correspondence and requests for materials should be addressed to

K.I. (itoh@nifs.ac.jp)

\section{New Thermodynamical Force in Plasma Phase Space that Controls Turbulence and Turbulent Transport}

\author{
Sanae-I. Itoh ${ }^{1,2} \&$ Kimitaka Itoh ${ }^{2,3}$
}

\begin{abstract}
${ }^{1}$ Research Institute for Applied Mechanics, Kyushu University, 6-1 Kasuga-Koen, Kasuga-city, 816-8580, Japan, ${ }^{2}$ Itoh Research Center for Plasma Turbulence, Kyushu University, Kasuga, 81 6-8580 Japan, ${ }^{3}$ National Institute for Fusion Science, 322-6, Oroshi-cho, Toki-city, 509-5292, Japan.
\end{abstract}

Physics of turbulence and turbulent transport has been developed on the central dogma that spatial gradients constitute the controlling parameters, such as Reynolds number and Rayleigh number. Recent experiments with the nonequilibrium plasmas in magnetic confinement devices, however, have shown that the turbulence and transport change much faster than global parameters, after an abrupt change of heating power. Here we propose a theory of turbulence in inhomogeneous magnetized plasmas, showing that the heating power directly influences the turbulence. New mechanism, that an external source couples with plasma fluctuations in phase space so as to affect turbulence, is investigated. A new thermodynamical force in phase-space, i.e., the derivative of heating power by plasma pressure, plays the role of new control parameter, in addition to spatial gradients. Following the change of turbulence, turbulent transport is modified accordingly. The condition under which this new effect can be observed is also evaluated.

 urbulence and turbulent transport have been subject to intensive studies as a central issue in the modern physics and science. The physics of turbulence and turbulent transport has been developed on the central dogma that the spatial gradients constitute the controlling parameters, such as Reynolds number and Rayleigh number ${ }^{1}$. This has also been the case in the research of turbulence in magnetized plasmas, and it has lead to the formalism that the transport fluxes are expressed in terms of transport matrix and spatial derivatives of mean variables ${ }^{2}$. However, the counter examples, which indicate that additional parameters (in addition to spatial gradients of global parameters) seem to strongly influence turbulence, have also been reported in the history of plasma turbulence research. For instance, the observations of dynamic responses against the sudden change of heating power have indicated limitations of transport pictures, which are based upon spatial gradients of mean parameters $^{3-5}$. Recent observations on the nonequilibrium plasmas in toroidal magnetic confinement device have unambiguously shown that the turbulence and transport change much faster than global parameters, after an abrupt (step-function-like) change of heating power ${ }^{3}$. This observation has induced the hypothesis, that the heating power directly influences the turbulence and transport, which has long been suggested (though less decisively) by many experiments (experimental reports include, e.g., refs. 4 and 5).

Here we propose a theory of turbulence in inhomogeneous magnetized plasmas, showing that the heating power directly influences the turbulence. New mechanism, that an external source couples with plasma fluctuations in phase space so as to affect turbulence, is investigated. A new thermodynamical force in phase-space, i.e., the derivative of heating power by plasma pressure, plays the role of new control parameter, in addition to spatial gradients. Following the change of turbulence, turbulent transport is modified accordingly. This article provides a theoretical logic for describing the direct influence of heating power as dynamical force, in addition to spatial gradient of global parameters, on turbulence and turbulent transport. It is shown theoretically that

1. The plasma heating power directly influences the turbulence, without waiting the change of plasma parameters and their spatial gradients. This effect (i.e., immediate impact) is more effective for fluctuations with long-wave lengths.

2. New control parameter, $\left[\partial P_{\text {heat }} / \partial p\right] a^{2} / \chi_{N}$, is analogous to the Reynolds number which is the inhomogeneitydriven rate of change' normalized to diffusion rate, but is novel in the way that the thermodynamical force is the rate of change in velocity space (not in real space). (Here, $P_{\text {heat }}$ is the heating power density, $p$ is the plasma pressure, $a$ is the plasma radius (characteristic scale length of spatial gradient), and $\chi_{\mathrm{N}}$ is the turbulent thermal diffusivity. 
3. Accordingly, the turbulent transport increases when the heating power is switched on, if $\partial P_{\text {heat }} / \partial p>0$.

4. The condition under which this new effect can be observed is also evaluated.

\section{Results}

Model. The objective of the article is to introduce a theoretical logic for describing a direct influence of heating power as dynamical force, in addition to spatial gradient of global parameters, on turbulence and turbulent transport. The essence of the new mechanism that affects turbulence and turbulent transport in plasmas is illustrated by observing the kinetic equation,

$$
\left(\frac{\partial}{\partial t}+\boldsymbol{v} \cdot \nabla+\frac{e_{s}}{m_{s}}(\boldsymbol{E}+\boldsymbol{v} \times \boldsymbol{B}) \cdot \nabla_{\boldsymbol{v}}\right) f_{s}(\boldsymbol{v}: t)=S+C,
$$

where $\boldsymbol{x}$ and $\boldsymbol{v}$ denote the spatial and velocity coordinates, $f, S, C$ are the distribution function, source and collision operator, respectively ${ }^{6}$. (The suffix $s$ indicates the particle species.) In order to study the evolution of turbulence, the distribution function is separated into the mean and perturbation, as $f=f_{0}+\tilde{f}$, and the evolution of $\tilde{f}$ is investigated, where the symbol $\sim$ indicates the fluctuation part. The source in the phase space $S$ includes the particle, momentum and energy supplies, is a functional of the distribution function, and depends on phase space variables as well as on time owing to the conditions of external circuit, so that it is expressed as $S[f ; \boldsymbol{v}, \boldsymbol{x}, t]$. Therefore, it naturally contains the component, which is coherent to the fluctuation of interest,

$$
S[f ; \boldsymbol{v}, \boldsymbol{x}, t]=S\left[f_{0}+\tilde{f} ; \boldsymbol{v}, \boldsymbol{x}, t\right]=S_{0}+\frac{\partial S\left[f_{0} ; \boldsymbol{v}, \boldsymbol{x}, t\right]}{\partial f_{0}} \tilde{f} .
$$

Thus the fluctuating part of equation (1a) takes a from, for electrostatic perturbations,

$$
\begin{aligned}
& \left(\frac{\partial}{\partial t}+\boldsymbol{v} \cdot \nabla+\frac{e_{s}}{m_{s}}(\boldsymbol{v} \times \boldsymbol{B}) \cdot \nabla_{v}\right) \tilde{f}(\boldsymbol{v}: t)=-\frac{e_{s}}{m_{s}} \tilde{\boldsymbol{E}} \cdot \nabla_{\boldsymbol{v}} f_{0}+ \\
& \frac{\partial S\left[f_{0} ; \boldsymbol{v}, x, t\right]}{\partial f_{0}} \tilde{f}+\tilde{C} .
\end{aligned}
$$

The RHS of equation (1c) describes the source and sink of perturbation. The first term indicates the driving mechanisms owing to the plasma inhomogeneity, and the second term shows a new mechanism analyzed in this article. This new term modifies the response timescale in changing the phase-space structure of $f$. It is well known that the externally-induced change in $f$ contains not only the slow change of $f$ in the whole velocity-space but also the rapid and localized changes in phase space. Therefore, the second term in the RHS represents the change rate of distribution function by heating (as the cause of structure change), and it directly couples with and affects the fluctuations. This term jumps at the on/off of heating, so that the on/ off of heating can immediately influence the fluctuation dynamics, without waiting the slower change of the mean $f_{0}$.

In order to examine the impact of the $2^{\text {nd }}$ term in RHS of equation (1c) on turbulence, we employ fluid-like equations in describing the turbulence in magnetically-confined inhomogeneous plasmas. (The hierarchy of model equations that describe plasma turbulence has been explained in, e.g., refs. 6 and 7.) Following the literature, we take electrostatic potential, $\phi$, plasma current along the magnetic field line, $J$, and pressure, $p$, as relevant parameters. We study here the case, where the external heating source, $P_{\text {heat }}(x, t)$, depends on plasma parameters. Being stimulated by the experiments in ref.3, where the absorption power via electron cyclotron resonance heating $(\mathrm{ECH})$ depends on the local density and energy of trapped electrons in phase space, etc., we simply choose the case that the deposition power is sensitive to $p$ (as in ref.8),

$$
P_{\text {heat }}(x, t)=\overline{P_{\text {heat }}(x, t)}+\frac{\partial P_{\text {heat }}}{\partial p} \tilde{p}+\cdots
$$

where $\overline{P_{\text {heat }}(x, t)}$ is an averaged heating power, and $\left(\partial P_{\text {heat }} / \partial p\right) \tilde{p}$ is the modulation of heating power which is induced by the presence of the fluctuations, that corresponds to the $2^{\text {nd }}$ term in RHS of equation (1c). Equation (2) has a simplification in two aspects. First, the perturbation of $P_{\text {heat }}(x, t)$ can also drive perturbations in $\phi$ and $J$. Second, other sources (particle source, electric induction, etc.) should be considered as well. Leaving these additional effects to future thorough studies, we take here a simplification for the analytic transparency of the argument.

By use of a method of the dressed test mode, the dynamical equation of fluctuations in magnetized turbulent plasmas has been derived, to which the effect of heating, equation (2), is introduced, as

$$
\left\{\partial_{\mathrm{T}}+\boldsymbol{L}\right\} \tilde{\boldsymbol{f}}=\left(0,0,\left(\partial P_{\text {heat }} / \partial p\right) \tilde{p}\right)^{\mathrm{T}},
$$

where $\tilde{f}=(\phi, J, p)^{\mathrm{T}}$ denotes the fluctuating fields, $L$ is the renormalized operator, which includes the linear instability mechanisms and the decorrelation by ambient turbulence ${ }^{8,9}$. Abbreviating as $F \equiv$ $\partial P_{\text {heat }} / \partial p$, equation ( $3 a$ ) is rewritten as

$$
\left\{\partial_{\mathrm{T}}+\overline{\boldsymbol{L}}\right\} \tilde{\boldsymbol{f}}=0
$$

where $\bar{L}_{3,3}=L_{3,3}-F$ and $\bar{L}_{i, j}=L_{i, j}$ otherwise. The screened operator, which includes the effects of this direct heating power, is given as

$$
\boldsymbol{L}=\left(\begin{array}{ccc}
\bar{\mu} \nabla_{\perp}^{4} & \nabla_{\|} & (\boldsymbol{b} \times \kappa) \cdot \nabla \\
\xi \nabla_{\|} & \xi \eta_{\|}-\xi \bar{\lambda} \nabla_{\perp}^{2} & 0 \\
\left(\nabla p_{0} \times \boldsymbol{b}\right) \cdot \nabla & 0 & -\bar{\chi} \nabla_{\perp}^{2}-F
\end{array}\right)
$$

with

$$
\bar{\mu}=\mu_{N}+\mu_{c}, \quad \bar{\lambda}=\lambda_{N}+\lambda_{c}, \quad \bar{\chi}=\chi_{N}+\chi_{c},
$$

where $\boldsymbol{b}$ is the unit vector in the direction of mean magnetic field, $\mathbf{\kappa}$ is the curvature of magnetic field, $\nabla p_{0}$ denotes the mean pressure gradient, operators $\nabla_{\|}$and $\nabla \perp$ denote the derivatives parallel to and perpendicular to the main magnetic field, respectively, $\mu, \eta_{\|}$, $\lambda$, and $\chi$ stand for viscosity, electric resistivity, current diffusivity and thermal conductivity, respectively. The coefficient $\xi$ is defined as $\xi=a^{2} \delta_{c}^{-2}$, where $a$ is a minor radius of torus (typical global scale length), and $\delta_{c}$ is the collisionless skin depth. The suffix $\mathrm{c}$ and $\mathrm{N}$ denote the contributions by molecular dissipation effects and ambient turbulence (for which explicit forms are given in ref.9), respectively. The scale-invariant property of this pressure-gradient-driven turbulence (in the limit of $F=0$ ) was explained in ref.10. As in the literature, all quantities are normalized ${ }^{9}$.

Effects on Microscopic Turbulence and Turbulent Transport. For the given intensity of ambient turbulence, equation (3) predicts that strong instabilities can occur for microscopic fluctuations (with larger wave number) and that fluctuations with longer wavelengths are less unstable (or stable). The state of self-sustained turbulence, where the nonlinearly-destabilizing and -stabilizing mechanisms balance, has been derived. Replacing the term $\bar{\chi}=\chi_{N}+\chi_{c}$ in literature ${ }^{9}$ by $\bar{\chi}=\chi_{N}+\chi_{c}-F k_{\perp}^{-2}$, the nonlinear marginal stability condition is derived as

$$
\Im=\Im_{c}\left(k_{y}\right),
$$

where the LHS of Eq.(5a) is given in terms of the renormalized turbulent transport coefficients as 


$$
\Im \equiv \frac{G_{0}}{s^{4 / 3}} \frac{\left(\lambda_{N}+\lambda_{c}\right)^{2 / 3}}{\left(\chi_{N}+\chi_{c}-F k_{\perp}^{-2}\right)\left(\mu_{N}+\mu_{c}\right)^{1 / 3}} .
$$

and the RHS of Eq.(5a) takes the minimum value $\Im_{c}^{*}=1.67$ at the characteristic mode number $k^{*}=\sqrt{b^{*}}\left(s^{2} / \lambda_{N} \mu_{N}\right)^{1 / 6}$ with $b^{*}=0.43$. Here, $G_{0}=\kappa p_{0}^{\prime}$, denotes the driving parameter and $s$ stands for the magnetic shear parameter ${ }^{2}$, both of which represent the spatial gradient of global plasma parameters. In a dimensional form, Eq.(5b) is rewritten as

$$
\Im=\alpha \frac{\kappa p_{0}^{\prime} \tau_{A p}^{2 / 3}}{m_{i} n_{0}} \frac{a^{8 / 3}}{\left(\chi-F k_{\perp}^{-2}\right) \mu^{1 / 3}},
$$

where $\alpha=s^{-4 / 3} \xi^{-2 / 3}$ is a parameter which is independent of gradient, $\mathrm{m}_{\mathrm{i}} \mathrm{n}_{0}$ is the plasma mass density, $\tau_{\mathrm{Ap}}$ is the transit time of Alfven wave at macro-scale of plasma majour radius $\mathrm{R}, \tau_{A p}=R$ $\sqrt{\mu_{0} m_{i} n_{0}} B_{0}^{-1}$. As is the case of Rayleigh number, the competition between the pressure gradient and diffusive damping by thermal conductivity and viscosity is apparent in Eq.(5c).

By use of the minimum value of $\Im, \Im_{c}^{*}=1.67$, one has the relation equation (in the limit of small collisional dissipations) as

$$
\left(\chi_{N}-F k_{\perp}^{-2}\right) \chi_{N}^{-1 / 3} \equiv \frac{1}{\Im_{c}^{*}} \frac{G_{0}}{s^{4 / 3}} \frac{\lambda_{N}^{2 / 3}}{\mu_{N}^{1 / 3} \chi_{N}^{1 / 3}} .
$$

One has two limiting solutions from equation (6):

$$
\chi_{N} \simeq \chi_{N, 0}+F k_{\perp}^{-2}+\cdots \quad \text { for } F \ll \chi_{N, 0} k_{\perp}^{2}
$$

and

$$
\chi_{N} \simeq F k_{\perp}^{-2}+\cdots \text { for } F \gg \chi_{N, 0} k_{\perp}^{2},
$$

where $\chi_{N, 0}$ is turbulent thermal conductivity in the absence of heat sources,

$$
\chi_{N, 0} \equiv \frac{1}{\Im_{c}^{* 3 / 2}} \frac{G_{0}^{3 / 2}}{s^{2}} \frac{\lambda_{N}}{\mu_{N}^{1 / 2} \chi_{N}^{1 / 2}} .
$$

Equation (7) illustrates the competition between the impact of plasma heating $(\operatorname{term} F)$ and diffusive transport $\chi_{N, 0} k_{\perp}^{2}$. Thus, the impact of heating on fluctuation is much stronger for modes with longer correlation lengths.

Effects on Long-Range Fluctuations. The long-range fluctuations, the radial correlation length of which is of the order of plasma size, are much more stable than microscopic perturbations, but can be excited to finite amplitude and sustained by nonlinear fluctuating force ${ }^{11}$. Such nonlinearly driven fluctuations can have substantial influence on turbulent transport ${ }^{12}$. By solving the stochastic equation, the amplitude of nonlinearly-driven long-range fluctuations has been evaluated ${ }^{11}$. The influence of heating power on it is discussed here. A stochastic equation has been derived as ${ }^{13-15}$

$$
\left\{\partial_{T}+\bar{L}\right\} f=\tilde{\sigma}
$$

where $\tilde{\sigma}=\left(\tilde{\sigma}_{1}, \tilde{\sigma}_{2}, \tilde{\sigma}_{3}\right)^{T}$ is the nonlinear fluctuating force acting on the fluctuation of interest ${ }^{16}$. The coherent effect of heating is included in $\bar{L}$ (say, $\bar{L}_{33}$ ). (In equation (3b), the fluctuating force is neglected, because the self-sustained turbulence develops without introducing the effect of nonlinear fluctuating force.)

Following the standard procedures, the statistical average of amplitude of long-range fluctuations is calculated. Following ref.15, the eigenvalue of the matrix $\overline{\boldsymbol{L}},-\Gamma_{m}\left(m=1,2,3\right.$ and $\left.\Gamma_{1}<\Gamma_{2}<\Gamma_{3}\right)$, the corresponding eigenvectors, $\boldsymbol{e}_{m}$, and the projection matrix to each eigenvector, $\boldsymbol{A}_{m}$, are introduced. (The expression of the projection matrix $\boldsymbol{A}_{m}$ is given in ref.15 and is not reproduced here). The leaststable element $m=1$ is chosen, and more stable components, which have larger damping rates, are neglected. By use of the projections of the fluctuations and source onto the least stable components, $\varphi_{1}=$ $\boldsymbol{e}_{1} \cdot \boldsymbol{A}_{1} \boldsymbol{f}$ and $\hat{\sigma}_{1}(t)=\boldsymbol{e}_{1} \cdot \boldsymbol{A}_{1} \tilde{\sigma}$, equation (9) is simplified as $(\partial / \partial t+$ $\left.\Gamma_{1}\right) \varphi_{1}=\hat{\sigma}_{1}(t)$. The solution of the least stable element yields a statistically-averaged value as

$$
\left\langle\varphi_{1} \varphi_{1}\right\rangle=\frac{1}{2 \Gamma_{1}}\left\langle\hat{\sigma}_{1}(t) \hat{\sigma}_{1}(t)\right\rangle,
$$

where $\langle\cdots\rangle$ indicates the long-time average. In obtaining equation (10), $\tilde{\sigma}$ is modelled as Gaussian white noises, $\left\langle\tilde{\sigma}_{i}(\tau) \tilde{\sigma}_{j}\left(\tau^{\prime}\right)\right\rangle \propto$ $\delta\left(\tau-\tau^{\prime}\right)$, because it has a short auto-correlation time. In principle, $\left\langle\tilde{\sigma}_{i}(\tau) \tilde{\sigma}_{j}\left(\tau^{\prime}\right)\right\rangle$ has a short but finite correlation time, but this does not change the conclusion of the analysis ${ }^{14,15}$.

The eigenvalue of current-diffusive interchange mode (the instability described by the system of equation (3)) is determined as equation (34) of ref.17. Away from the linear stability limit, the decorrelation rate was given as $\Gamma_{1}=\chi_{N} k_{\theta}^{2}$. Replacing $\bar{\chi}=\chi_{N}+\chi_{c}$ in literature by $\bar{\chi}=\chi_{N}+\chi_{c}-F k_{\perp}^{-2}$ in the presence of heating power here, we have the decorrelation rate for the long-range mode as

$$
\Gamma_{1}=\chi_{\mathrm{N}} k_{\theta}^{2}-F k_{\theta}^{2} / k_{\perp}^{2},
$$

where contribution of molecular diffusivity is neglected. The nonlinear noise source $\left\langle\hat{\sigma}_{1} \hat{\sigma}_{1}\right\rangle$ is induced by microscopic fluctuations ${ }^{11}$, which are less sensitive to heating power than the long-range fluctuations. From equations (10) and (11), the mean amplitude of the linearly-stable global mode in the turbulent plasma is given as

$$
\left\langle\varphi_{1} \varphi_{1}\right\rangle=\frac{1}{1-F \chi_{\mathrm{N}}^{-1} k_{\perp}^{-2}}\left\langle\varphi_{1} \varphi_{1}\right\rangle_{0}
$$

where $\left\langle\varphi_{1} \varphi_{1}\right\rangle_{0}$ is the intensity in the absence of the effect of the heating, and an estimate $\chi_{\mathrm{N}} k_{\perp}^{2} \simeq 2 \chi_{\mathrm{N}} k_{\theta}^{2}$ is employed.

From this result, we see that an enhancement of the long-range fluctuation is prominent if $F / \chi_{\mathrm{N}} k_{\perp}^{2} \rightarrow 1$. That is, rewriting the wavelength in terms of the global scale length, $k_{\perp}^{-1} \sim a$, the condition for the strong influence of the heating power on fluctuations is written as

$$
\frac{\partial P_{\text {heat }}}{\partial p} \frac{a^{2}}{\chi_{\mathrm{N}}} \rightarrow 1
$$

The LHS is the ratio between the new time rate associated with the plasma heating power, $\partial P_{\text {heat }} / \partial p$, and the decorrelation rate owing to the turbulent diffusion. The new control parameter, $\left[\partial P_{\text {heat }} / \partial p\right] \mathrm{a}^{2} /$ $\chi_{N}$, is in one hand analogous to the Reynolds number which is the 'inhomogeneity-driven rate of change' normalized to decorrelation rate by diffusion. It is novel because the thermodynamical force $\partial P_{\text {heat }} / \partial p$ is the rate of change not in real space but in velocity space. See the comparison in Table 1 . The value $\left[\partial P_{\text {heat }} / \partial p\right] \mathrm{a}^{2} / \chi_{\mathrm{N}}$ can be $\mathrm{O}(1)$ in the experimental condition of ref.3, if one uses an estimate $\partial P_{\text {heat }} / \partial p \simeq P_{\text {heat }} / p$.

We next study the response of the long-range fluctuation after the onset of the heating power. Consider the case that the strong heating is turned on at $t=t_{0}$, and the tem $F$ is given as $F=F H\left(t-t_{0}\right)$, where $H\left(t-t_{0}\right)$ is a Heviside function. The statistical average of fluctuation intensity is deduced from the stochastic equation. The memory of the state before the onset of heating is lost at the rate $\chi_{\mathrm{N}} k_{\perp}^{2}$, and the access to the new stationary value takes place at the rate of $\chi_{\mathrm{N}} k_{\perp}^{2}-F$.

\section{Discussion}

This article has shown a theoretical framework that the heating power can couple to and influence the turbulence directly and immediately (i.e., without the change of mean parameters). The understanding of this new direct link between heating source and turbulence has strong impact on our scientific predictability of 
Table 1 | Comparison between control parameters in gradient-driven turbulences and the new parameter associated with plasma heating. In Reynolds number and Rayleigh number, $U / a$ and $\Delta T / a$ represent the spatial gradients of global parameter, $v$ is the kinematic viscosity of fluid, $g$ is the acceleration of gravity and $\beta$ is the coefficient of thermal expansion

$\begin{array}{ll}\text { Sheared flow (Reynolds number) } & R_{e}=\frac{a U}{v} \rightarrow \frac{U}{a} \frac{a^{2}}{v} \\ \text { Fluid heated from bottom (Rayleigh number) } & R_{a}=\frac{a^{3} \beta g \Delta T}{v \chi} \rightarrow \frac{\Delta T}{a} \beta g \frac{a^{4}}{v \chi} \\ \begin{array}{l}\text { A pressure-gradient turbulence in } \\ \text { magnetized plasmas }\end{array} & \Im=\alpha \frac{\kappa p_{0}^{\prime} \tau_{A p}^{2 / 3}}{m_{i} n_{0}} \frac{a^{8 / 3}}{\chi \mu^{1 / 3}} \\ \begin{array}{l}\text { New control parameter associated with } \\ \text { plasma heating }\end{array} & \frac{\partial P_{\text {heat }}}{\partial p} \frac{a^{2}}{\chi}\end{array}$

dynamic response of turbulence in the system far from equilibrium that is sustained by external supply of energy, momentum, etc. This framework can provide possible explanations for the long-standing mysteries, that the transport looks to change very rapidly, when the plasma heating power changes abruptly $y^{3-5}$. For quantitative explanation of these phenomena, further theoretical analyses are required, along the line suggested in this article. First, an elaborate theory is to be developed in order to quantify the influence of heating in collisionless plasmas. For instance, the plasma heating via radio frequency waves can modify the distribution function of trapped particles $^{2}$ in phase space (trapped particles are limited in velocity space, and subject to restricted motion in real space), and such process can be quantified. An analysis on the growth rate of dissipativetrapped particle instability ${ }^{2}$ was performed ${ }^{18}$, and it was shown that the influence of this new mechanism is substantial for the low-frequency trapped particle modes to which the long-range fluctuation in ref. 12 was attributed. Namely, heating power is immediately transferred to the free energy source of the mode. The second issue is the so-called nonlocal problem. In reality, influence on microscopic turbulence can appear even in the case where the heating power is absorbed at distant location. The long-range interactions (via, e.g., fluctuations with long-correlation length ${ }^{19}$, streamers $^{20}$, spreading of turbulence ${ }^{21,22}$, etc.) are candidates of mechanisms that explain such non-local-in-space phenomena.

Analyses have been performed on 'whether the observations in ref.3 require new theoretical ideas, or can be explained within the existing theoretical framework for strongly-nonlinear processes and non-local-in-space processes (including the fluctuations with longcorrelation length and spreading of turbulence, or integral formulation of heat flux $\left.{ }^{23,24}\right)^{\prime}$.

The experimental findings are reported in ref.25 more thoroughly. The experimental study has shown that there are two distinct time scales in the evolution of plasmas after the on/off of heating power (which is absorbed at the plasma center); i.e., the time scale for the well-known slow evolution of mean plasma parameters (such as the temperature and temperature gradient) over the whole plasma, and the very fast reaction time in the heat flux and fluctuation intensity ${ }^{25}$. While the former is in the range of 40-100 ms, the latter is of the order of $1 \mathrm{~ms}$, the exact value of which is obscured by the time resolution of diagnostic system.

The test of various models has also been in progress ${ }^{25}$. The result is as follows. (1) The conventional way of modelling, in which the strong nonlinearity of gradient-flux relation can cause a rapid response after on/off of heating, is far from satisfactory (as was in the case of rapid response after the transition of confinement property at the edge ${ }^{26}$ ). (2) The integral formulation (in which the heat flux is expressed in terms of spatial integral of gradients, and belongs to the class of the non-local-in-space models) was tested. This framework can explain some limited cases of experiments (where modulation amplitude of heating power is small), but has failed to explain the difference of the two time scales, as reported in ref.25. (3) Although it is considered that the mechanisms based on the fluctuations with long-correlation length and on the spreading of turbulence might work in the present experiment, the existing theoretical framework alone seems insufficient: This is because, within the existing framework, the statistical-average property of these fluctuations (the fluctuations with long-correlation length and spreading of turbulence) is correlated with mean plasma parameters, so that the sudden variation of these activities without a change of global parameters and gradients has not yet been explained. Thus, while the firm conclusion whether the previously-published theoretical framework is sufficient or not waits future detailed measurements of fluctuation dynamics, it is highly plausible that the observation in ref.3 can not be explained by existing models alone. The new theoretical framework in this article explains the emergence of two time scales (i.e., the rapid response of flux and fluctuation intensity right after the on/off of heating power, as well as the slow time scale of changes of global parameters and gradients). The unification of this new idea and existing theoretical framework provides a key to understand the experimental observations that the turbulence and transport change much faster than the change of global parameters.

\section{Methods}

The renormalization method that leads to equation (4a) is explained in Chapter 8 of ref.9 in detail. The deduction of equation (5) from equation (4) is explained in Chapter 9 of ref.9.

1. Rayleigh, J. W. S. On the convective currents in a horizontal layer of fluid when the higher temperature is on the under side, Phil. Mag. 32 529-546 (1916).

2. Wesson, J. A. Tokamaks. (Oxford, 1987).

3. Inagaki, S. et al. Heating power as novel controll parameter for turbulence and turbulent transport. submitted to Phys. Rev. Lett. (2012).

4. Luce, T. C., Petty, C. C. \& de Haas, J. C. M. Inward energy transport in tokamak plamsas. Phys. Rev. Lett. 68, 52-55 (1992).

5. Stroth, U., Giannone, L., Hartfuss \& H.-J. the ECH Group \& the W7-AS Team, Fast transport changes and power degradation in the W7-AS stellarator. Plasma Phys. Control. Fusion 38, 611-618 (1996).

6. Balescu, R. Aspects of Anomalous Transport in Plasmas. (IoP, 2005).

7. Diamond, P. H., Itoh, S.-I. \& Itoh, K. Modern Plasma Physics Vol.1, Physical Kinetics of Turbulent Plasmas. (Cambridge University Press, 2010).

8. Itoh, K., Yagi, M., Itoh, S.-I. \& Fukuyama, A. On turbulent transport in burning plasmas. Plasma Phys. Contr. Fusion 42, 855-861 (2000).

9. Itoh, K., Itoh, S.-I. \& Fukuyama, A. Transport and Structural Formation in Plasmas (IoP, 1999).

10. Connor, J. W. Pressure gradient turbulent transport and collisionless reconnection. Plasma Phys. Control. Fusion 35, 757-763 (1993).

11. Itoh, S.-I. \& Itoh, K. Statistical theory and transition in multiple-scale-length turbulence in plasmas. Plasma Phys. Control. Fusion 43, 1055-1102 (2001).

12. Inagaki, S. et al. Observation of long-distance radial correlation in toroidal plasma turbulence. Phys. Rev. Lett. 107, 115001-1-115001-5 (2011).

13. Krommes, J. A. Recent results on analytical plasma turbulence theory: realizability, intermittency, submarginal turbulence and self-organized criticality. Plasma Phys. Control. Fusion 41, A641-A652 (1999).

14. Itoh, S.-I. \& Itoh, K. Statistical theory of subcritically-excited strong turbulence in inhomogeneous plasmas. I. J. Phys. Soc. Jpn. 68, 1891-1906 (1999).

15. Itoh, S.-I. \& Itoh, K. Statistical theory of subcritically-excited strong turbulence in inhomogeneous plasmas. II. J. Phys. Soc. Jpn. 68, 2611-2625 (1999).

16. Mori, H. Transport, collective motion, and Brownian motion. Prog. Theor. Phys. 33, 423-455 (1965).

17. Itoh, K., Ichiguchi, K. \& Itoh, S.-I. Beta limit of resistive plasma in a heliotron. Phys. Fluids B 4, 2929-2933 (1992).

18. Itoh, S.-I., \& Itoh, K. Immediate Influence of Heating Power on Turbulent Plasma Transport, 24th IAEA Fusion Energy Conference (San Diego, 2012), IAEA CN197/PD/P8-11.

19. Itoh, K., Itoh, S.-I., Yagi, M. \& Fukuyama, A. Seesaw mechanism in turbulencesuppression by zonal flows. J. Plasma Fusion Res. Series 8, 119-121 (2009).

20. Yamada, T. et al. Anatomy of plasma turbulence. Nature Phys. 4, 721-725 (2008).

21. Diamond, P. H. \& Diamond, P. H. \& Hahm, T. S. Phys. Plasmas 2, 3640-3649 (1995)

22. Hahm, T. S., Diamond, P. H., Lin, Z., Itoh, K. \& Itoh, S.-I. Turbulence spreading into the linearly stable zone and transport scaling. Plasma Phys. Control. Fusion 46, A323-A333 (2004) 
23. Iwasaki, T. et al. Non-local model analysis of heat pulse propagation and simulation of experiments in W7-AS. J. Phys. Soc. Japan 68, 478-486 (1999)

24. Milligen, B. Ph. van, Sanchez, R., \& Carreras, B.A. Probabilistic finite-size transport models for fusion, Anomalous transport and scaling laws. Phys. Plasmas 11, 2272-2285 (2004).

25. Inagaki, S. et al. Is Turbulence Determined by Local Temperature Gradient?, 24th IAEA Fusion Energy Conference (San Diego, 2012), IAEA CN-197/EX/10-1

26. Cordey, J. G. et al. The time behavior of the thermal conductivity during $\mathrm{L}-\mathrm{H}$ and H - L transitions in JET. Plasma Phys. Control. Fusion 36 A267-A272 (1994).

\section{Acknowledgement}

We thank Dr. S. Inagaki, Prof. A. Fukuyama, Prof. A. Fujisawa, Prof. K. Ida, Prof. U. Stroth and Prof. P. H. Diamond for useful discussions. This work is partly supported by the Grant-in-Aid for Scientific Research of JSPS, Japan (21224014, 23244113), by the collaboration programs of the RIAM of Kyushu University and of NIFS (NIFS10KOAP023), and by Asada Science Foundation.

\section{Author contributions}

SII provides a problem definition, and KI led the theoretical deduction. All authors discussed the results and reviewed the manuscript.

\section{Additional information}

Competing financial interests: The authors declare no competing financial interests.

License: This work is licensed under a Creative Commons

Attribution-NonCommercial-NoDerivative Works 3.0 Unported License. To view a copy of this license, visit http://creativecommons.org/licenses/by-nc-nd/3.0/

How to cite this article: Itoh, S.-I. \& Itoh, K. New Thermodynamical Force in Plasma Phase Space that Controls Turbulence and Turbulent Transport. Sci. Rep. 2, 860; DOI:10.1038/ srep00860 (2012). 